\title{
Sonochemical Reformatsky Reaction Using Indium ${ }^{\dagger}$
}

\author{
Keukchan Bang, Kooyeon Lee, Yong Kwang Park, and Phil Ho Lee ${ }^{\star}$ \\ Department of (hemistry: Kangron Fational Lniversity, ('hunchon 200-701, Korea \\ Received leptember $+_{1} 2001$
}

\begin{abstract}
Sonochemical Refonnatsky reaction of aldehydes or ketones with ethyl bromoacetate in the presence of indium afforded $\beta$-hyydrosyesters in good to excellent yields under mild conditions. 2- or 3-Hyddroxy benzaldehyde that contains an acidic hydrogen reacted with ethyl bromoacetate to provide the desired compounds with the same efficiency. In the case of etlyl 2-bromopropanoate and ethyl 2-bromo-2-methylpropanoate, the desired products were obtained in good yields. Reaction of aldehyde with indium reagent in the presence of ketone group proceeded chemoselectively:
\end{abstract}

Key Words : Indium. Reformatsky reaction. $\beta$-Hydroxyester. Sonochemical reaction

\section{Introduction}

The Reformatsky reaction, which is regarded as one of the most fundamental reactions in C-C bond formations. is the reaction of a carbonyl compound with an $\alpha$-halocster in the presence of zinc metal to furnish $\beta$-hydroxyesters.' The products are one of the most important intermediates in organic synthesis. - Recently. scicral modificd Relormatsky reactions using other metals have been reported." Also. the scope of the Reformatsky reaction has been cxicnded with special techniques for the activation of the metal (e.g. for removal of the oxide layer. and the preparation of linely dispersed metal). ${ }^{2}$ The activation of zinc by treatment with iodine or dibromomethane. or washing with dilute hydrochloric acid prior to use. is only moderately successlul in the most known procedures. Much more eftective protocol is the usc of special alloys - e.g. Zn-Cu couplc. or the reduction of zinc halides using polassium (the so-called Ricke procedurc ${ }^{5}$ ) or potassium graphitc. ${ }^{+}$Although the application of ultrasound has been reported for zine metal. promoters such as iodine and potassium iodide were necded somelimes to oblain the desired products in good yiclds. ${ }^{6}$ Our interests in extending the scope of the Reformatsky reaction and subsequent application of indium metal to moden organic synthesis ${ }^{7}$ have led us to investigate indiun mediated Reformatsky reaction. As part of our continuing cflton to cxpand the synthetic utility of indium. we now report a sonochemical Reformatsky reaction by the reaction of aldehydes or ketones with elhyl bromoacelate in the presence of indium (Scheme 1).

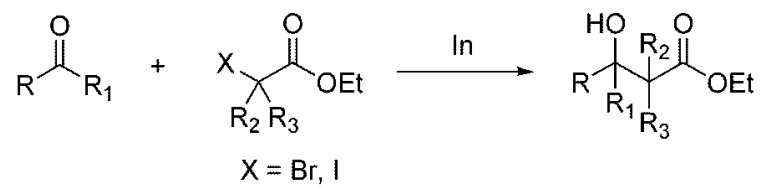

Scheme 1

This paper is dedicated to the deceased Protessor Sang Chul Shim for his outstanding achievements in organce chemistry.

\section{Results and Discussion}

To lind optimum conditions lor sonochemical Reformatsky reaction using indium. initially benzaldehyde reacted with chyyl bromoacetate in the presence of indium in various solvents. The results are summarized in Table I. Among the scicral solvents tested. THF gave the best results in terms of conversion and reaction time. The reaction of benzaldehyde with chyl bromoacetate in the presence of indium in THF altorded chyl 3-hydroxy-3-phenylpropanoate in $97 \%$ yicld (cntry 5). Sonication was carricd out at room tempcrature in a Fisher Scientific ultrasonic cleaner bath. which delivered a $43 \mathrm{kH} \%$ wave. with a fixed clectrical power of 435 Watts. However. the yiclds were decreased in other solyents under the identical conditions despite longer reaction time. Sonochemical reaction condition was supcrior to stirring condition. In the absence of sonic waves. the reaction occurred much more slowly $(17 \mathrm{~h}$ ) and the yicld of the $\beta$-hydroxyester

Table 1. Lixamination of optimum condition of Relomatshy reaction

\begin{tabular}{|c|c|c|c|}
\hline Futtry & Solvent & Time (h) & Isolated vield $(\%)^{4}$ \\
\hline 1 & DMF & 8 & 0 \\
\hline 2 & DMF & 4 & $22 r$ \\
\hline 3 & THF & 8 & 58 \\
\hline 4 & THF & 17 & 70 \\
\hline 5 & THF & 2 & $97^{\prime}$ \\
\hline 6 & THF & 2 & $97^{\prime s}$ \\
\hline 7 & wal-IIL" & 4 & $78^{\prime}$ \\
\hline 8 & $\mathrm{H}_{2} \mathrm{O}$ & 19 & 0 \\
\hline 9) & $\mathrm{H}_{2} \mathrm{O}$ & 4 & $\gamma$ \\
\hline 10 & $\mathrm{ITH} / \mathrm{II}_{2} \mathrm{OO}^{\prime}$ & 16 & 0 \\
\hline 11 & $\mathrm{ITH} / \mathrm{II}_{2} \mathrm{O}^{c}$ & 9 & 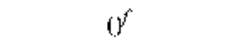 \\
\hline 12 & IIH $/ \mathrm{H}_{2} \mathrm{O}^{\prime}$ & 9 & $\gamma$ \\
\hline 1.3 & $\mathrm{MeOH} / \mathrm{HCl}^{\circ}$ & 17 & 0 \\
\hline
\end{tabular}

"The reaction mixture was stirred (rpm: ...900) unless ohenyise noted. "I $\mathrm{TH}$ was nol dried. "THF : $\mathrm{H}_{2} \mathrm{O}-1$ : 1. "I $\mathrm{THF}: \mathrm{H}_{2} \mathrm{O}-9: 1$. "MeOH : $\mathrm{HCl}-1: 4$. tultrasonic inadiation. E.thy.l indoacetate was uscd. 
Table 2. Preparation of $\beta$-hydroxyesters by In-mediated reactions of cabonyl compounds

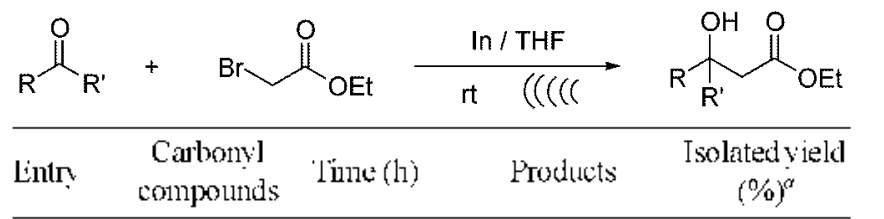<smiles>CCCC(=O)CC(=O)CC(O)CCc1ccccc1</smiles>

3<smiles>O=CCc1ccccc1</smiles><smiles>[V]</smiles><smiles>CCOC(=O)CC(O)Cc1ccccc1</smiles>

389
2.5

4<smiles>CC(C)C=O</smiles>

5<smiles>O=CC1CCCCC1</smiles>

2

6<smiles>O=Cc1ccccc1</smiles>
2

7<smiles>[Mg][Mg]</smiles><smiles>CCOC(=O)CC(O)c1ccccc1</smiles><smiles>O=CC(=[Co])c1ccccc1</smiles><smiles>CCOC(=O)CC(O)[10CH][14CH]</smiles>

9<smiles>O=C[Pb](Br)Br</smiles><smiles>CCOC(=O)CC(O)C([15CH])=[18O]</smiles>

9

91

10 (4-MeO)Ph $\stackrel{\mathrm{O}}{\mathrm{N}}_{\mathrm{H}} 2.5$<smiles>CCOC(=O)CC(O)c1ccccc1</smiles>

10

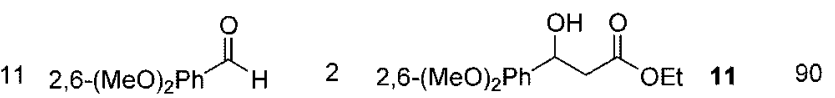

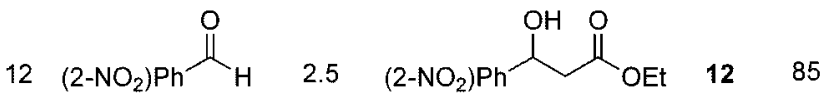

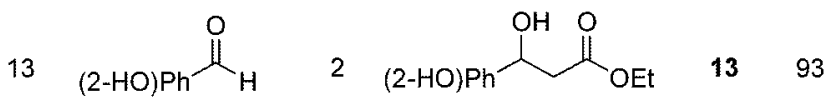

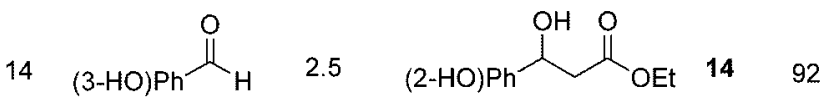

Table 2. Continued

Eintry

"Sonicalions were carried out at room temperature in a Fisher Scientibic ultrasonic cleaner bath, which delivered a $4.3 \mathrm{kHI}$. ware. with a fixed electrical power of 4.35 Watts. "Sonic wave was not used.

was only $70 \%$ (cntry 4). Rate per minute (rpm) for stirring conditions was $c a, 900$. Ethyl iodoacetate gave the similar result to chyl bromoacetate (entry 6). Also. organoindium reagent generated in situ from the reaction of I cquiv of indium with 1.5 cquiv of ethyl bromoacetate gave the best result. The use of indium less than I cquiy and cthyl bromoacetate less than 1.5 equiv resulted in sluggish reaction and gave lower yields as well as longer reaction time.

Table 2 summarized the experimental results and illustrates the efficiency and scope of the present method. Li the case of aliphatic aldelydes, the reaction afforded the desired compounds in excellent vields (entries $1 \sim 5$ ). For the aromatic aldehydes, the presence of various substituents. e.g. methyl (entry 7), chloro (entry 8). bromo (entry 9), monomethoxy (entry 10), dimethoxy (entry 11). or nitro (entry 12) on the aromatic ring showed little effects on the efficiency of the reactions. It should be mentioned that 2- or
23<smiles>CCOC(=O)C(C)(C)C(O)c1ccccc1</smiles>

$82 \%$

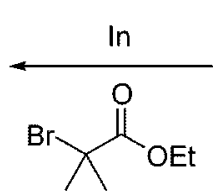

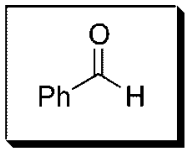

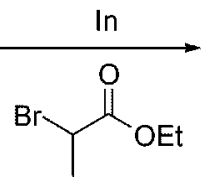<smiles>CCOC(=O)C(C)C(O)c1ccccc1</smiles>

$76 \%(d r=2.5: 1)$ 
3-hydroxyben/aldehyde that contains an acidic hydrogen reacted with ethyl bromoacclate to provide the desired compounds 13 and 14 in $93 \%$ and $92 \%$ yields. respectively. (cntries 13 and 14). In the case of 2-furaldehyde and 2thiophenecarboxaldehyde. $\beta$-hy droxyesters were oblained in good yields (cntries 15 and 16). Cimmamaldehyde was treated with ethyl bromoacelate to produce the desired compound in $93 \%$ yicld (entry 17). The prolocol developed here was also applied to reactions with ketone. For cxample. acctone and cyclohexanone reacted with ethyl bromoacetate to produce the desired compounds in $73 \%$ and $82 \%$ yiclds. respectively (entries 18 and 19 ). For the aromatic ketones. the treatment of acctophenone (cniry 20) and p-methoxyacctophenone (entry 2l) with ehyl bromoacelate gave the $\beta$ hydroxyesters 20 and 21 in $90 \%$ and $88 \%$ yiclds. respectively. However indium-promoted reaction of acctophenone with ethyl bromoacctate gave $\beta$-hydroxyester 20 in $40 \%$ vield withoul sonic wave. The sonicated mixture of zinc chyl bromoacelate. and acelophenone gave no addition product in the absence of iodine."

In the case of alkyl substituted ethyl bromoacctatc. the desired products were obtained in good to cxcellent yiclds (Scheme 2). Benzaldehyde reacted with cthyl 2-bromopropanoate and cthyl 2-bromo-2-methylpropanoate in the presence of indium to give $\beta$-hy droxyesters 22 and 23 in $76 \%$ (dr $=2.5 ; 1)$ and $82 \%$ vields. respectively.

To investigate chemoselectivity of aldehyde and ketone group. the mixture of benzaldelyde and acclophenone was treated with ethyl bromoacctate and indium to producc selectively ethy 13-hydroxy-3-pheny lpropanoatc (6) as major compound. The results are summarized in Table 3 .

In summary. sonochemical Reformatsky reaction using indium afforded $\beta$-hydroxyesters in high yiclds. There are scieral advantages of the method such as (a) the organoindium compound was prepared and used in silu: (b) it has a wide application for clongation of carbon chains: (c) in comparison to the use of zinc and tin. the reaction with indium was not required any promoter (iodine or potassium iodide): (d) the organoindium intermediates did not react with themselves in normal conditions: (e) It should be men-

Table 3. Competition reaction of benzaldehyde and acetophenone
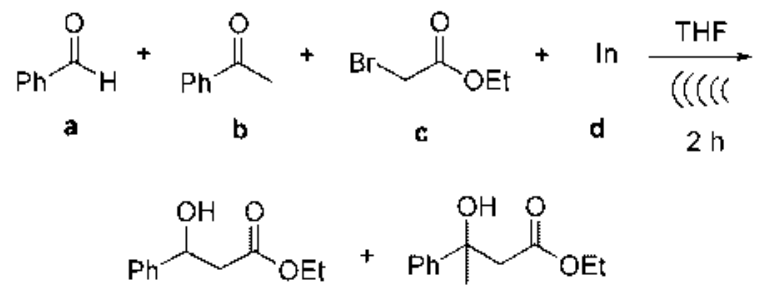

620

\begin{tabular}{|c|c|c|c|c|c|c|}
\hline \multirow{2}{*}{ Fintry } & \multicolumn{4}{|c|}{ Fquiv of reactanlts } & \multicolumn{2}{|c|}{ G.C ratio } \\
\hline & a & b & c & d & 6 & 20 \\
\hline l & 1.0 & 1.0 & 1.5 & 1.0 & 4.2 & I \\
\hline 2 & 1.0 & 1.0 & 1.0 & 1.0 & 237 & 1 \\
\hline 3 & 50 & 50 & 1.5 & 1.0 & 10.1 & 1 \\
\hline 4 & 50 & 50 & 1,0 & 1.0 & 10.1 & 1 \\
\hline
\end{tabular}

tioned that 2- or 3-hydroxybenzaldehyde that contains an acidic hỵdrogen reacted with ethyl bromoacetate to provide the desired compounds with the same clliciency: and (I) reaction of aldehyde with indium reagent in the presence of ketone group proceeded chemosclectively. The present method complements the existing synthetic methods of $\beta$-hydroxyester because of mild reaction condition and advantages of indium metal with regard to casy handling. high reactivity and sclectivity. low toxicity and operational simplicity

\section{Experimental Section}

The ${ }^{1} \mathrm{H}$ NMR and ${ }^{13} \mathrm{C}$ NMR spectra were recorded on Brucker JFS $48(400 \mathrm{MH} /$ ) spectrometer. Proton chemical shifts $(\delta)$ were reported in ppm downficld from tetramethylsilane (TMS), and '' $\mathrm{C}$ resonances were recorded using the 77.0 ppm $\mathrm{CDCl}_{3}$ resonance peak of the solycnt as internal relerence. Fouricr transform infrared (FTIR) spectra were recorded on a JASCO IR I0) spectrophotomeler. Mass spectra were obtained on a Autospec. M363 scrics. Column chromatography was perlormed on silica gel (Merck. 230-400 mesh). The gas cluromatograms were oblained on HP 5890 .

Typical procedure for indium-mediated sonochemical Reformatsky reaction: Ethỵl 3-hydiroxy-3-phenylpropanoate (6). To a solution of indium [indium powder (9).\%)\%) purchased from Aldrich Chem Co.: $58.0 \mathrm{mg} .0 .5 \mathrm{mmol}$ in THF (1.0 $\mathrm{mL}$ ) was added ethyl bromoacctatc (125.0 $\mathrm{mg}$. $0.75 \mathrm{mmol}$ ) and then benzaldchyde (53.0 mg. $0.5 \mathrm{mmol})$ under nitrogen at room temperature. After sonicating in a Fisher Scicntilic ultrasonic clcaner bath. which delivered a $43 \mathrm{kH} /$ wave with a fixed clectrical power of 435 Watts for 2 hr. the reaction mixture was quenched with saturated aqueous $\mathrm{NaHCO}_{3}$ solution. The aqucous layer was cxtracted with cther $(3 \times 25 \mathrm{~mL})$ and the combined organic layers were washed with water $(20 \mathrm{~mL})$ and brine $(20 \mathrm{~mL})$ and dricd with $\mathrm{MgSO}_{4}$. filtered and concentracted in vacto. The residuc was purificd by silica gel chromatography (hexanc : EtOAc $=5: 1$ ) leading to ethyl 3-hydroxy-3-pheny-lpropanoate $(94.0$ mig. 97\%). ${ }^{1} \mathrm{H}$ NMR (200 MHz. $\left.\mathrm{CDCl}_{3}\right) \delta 7.4-7.25(5 \mathrm{H} . \mathrm{m})$. $5.14(\mathrm{lH}, \mathrm{q} . J=4.0 \mathrm{~Hz}) .4 .19(2 \mathrm{H}, \mathrm{q} . J=7.20 \mathrm{~Hz}) .3 .14(1 \mathrm{H}$. br s). $2.83-2.64(2 \mathrm{H}, \mathrm{m}) .1 .26(3 \mathrm{H}, \mathrm{t} . J=7.00 \mathrm{~Hz}){ }^{1 .} \mathrm{C} \mathrm{CNMR}$ $\left(50 \mathrm{MHz}, \mathrm{CDCl}_{3}\right) \delta 172.64 .142 .62,128.69 .127 .94,125.81$. 70.40. 61.02. 43.41. 14.23: IR (filmi) 3460. 2980. 1720. 1490. $1450,1395.1370,1295.1260 \mathrm{~cm}^{-1}$ : MS (CI) calcd for $\mathrm{C}_{11} \mathrm{H}_{15} \mathrm{O}_{3}[\mathrm{M}+\mathrm{H}]^{-} 195$. found 195

Ethyl 3-hydroxyhexanoate (1) ' $\mathrm{H}$ NMR $(400 \mathrm{MHz}$. $\left.\mathrm{CDCl}_{3}\right) \delta 4.17(2 \mathrm{H.q.} J=7.15 \mathrm{~Hz}), 4.02(1 \mathrm{H} . \mathrm{ml}) .3 .01(1 \mathrm{H}$. d. $J=3.69 \mathrm{~Hz}) .2 .49(1 \mathrm{H}$. dd. $J=3.32 .3 .16 \mathrm{~Hz}) .2 .39(1 \mathrm{H}$. dd. $J=8.93,8.93 \mathrm{~Hz}) .1 .25-1.53(4 \mathrm{H}, \mathrm{ml}), 1.27(3 \mathrm{H} . \mathrm{t} . J=$ $7.20 \mathrm{~Hz}), 0.93(3 \mathrm{H} . \mathrm{t} . J=6.99 \mathrm{~Hz}):{ }^{13} \mathrm{C}$ NMR $(100 \mathrm{MHz}$. $\left.\mathrm{CDCl}_{3}\right) \delta 173.13 .67 .77 .60 .67 .41 .38,38.68,18.69 .14 .19$. 13.97: IR (film) 3480. 3050. 2960. 1720. $1260 \mathrm{~cm}^{-1}:$ MS (CI) calcd for $\mathrm{C}_{8} \mathrm{H}_{17} \mathrm{O}_{3}[\mathrm{M}+\mathrm{H}]^{-}$161. found 161 .

Ethyl 3-hydroxy-5-phenylpentanoate (2) ${ }^{1} \mathrm{H}$ NMR (400 $\mathrm{MHz}_{2} \mathrm{CDCl}_{3}$ ) $\delta 7.30-7.25$ (m. 2H). $7.21-7.16$ (nl. 3H). 4.17 $(\mathrm{q} . J=7.15 \mathrm{~Hz} .2 \mathrm{H}), 4.02(\mathrm{~m}, 1 \mathrm{H}) .3 .07(\mathrm{~d} . J=3.96 \mathrm{~Hz} .1 \mathrm{H})$, 2.82-2.78 (m, 1H). 2.73-2.68 (m, 2H). 1.89-1.82 (1m, 1H). 
$1.75-1.72(\mathrm{~m}, \mathrm{lH}), 1.26(1, J=7.08 \mathrm{H} z .3 \mathrm{H}) \cdot{ }^{13} \mathrm{C} \mathrm{NMR}(100$ $\left.\mathrm{MH} z, \mathrm{CDCl}_{3}\right) \delta 174.19,142.91,129.63,129.59,127.06$. 68.38, 61.90, 42.46, 39.28, 32.93, 15.34: IR (film) 3420. 2920. 1710, 1600, 1490, 1445,1360,1295, $1245 \mathrm{~cm}^{-1}$ : MS (Cl) calcd for $\mathrm{C}_{13} \mathrm{H}_{18} \mathrm{O}_{3}[\mathrm{M}+\mathrm{H}]^{\prime} 223$. found 223 .

Ethyl 3-hydroxy-t-phenylbutanoate (3) 'H NMR (400) $\left.\mathrm{MH} \% \mathrm{CDCl}_{3}\right) \delta 7.30(\mathrm{~m}, 2 \mathrm{H}), 7.22(\mathrm{~m}, 3 \mathrm{H}) .4 .26(\mathrm{~m}, \mathrm{lH})$. $4.13(\mathrm{q} . J=4.73 \mathrm{H} z .2 \mathrm{H}) .2 .98(\mathrm{~d} . J=3.86 \mathrm{H} z . \mathrm{lH}) .2 .85(\mathrm{q}$. $J=6.89 \mathrm{H} \% \mathrm{lH}) 2.76(\mathrm{q} . J=6.56 \mathrm{H} / \mathrm{lH}) .2 .39-2.25(\mathrm{~m}$. $2 \mathrm{H}) .1 .25(1 . J=7.16 \mathrm{~Hz} .3 \mathrm{H}):{ }^{13} \mathrm{C} \mathrm{NMR}\left(100 \mathrm{MH} / . \mathrm{CDCl}_{3}\right)$ $\delta 172.71,137.71,129,45,128.53,126,60,69,05,60.71$. 42.95.40.54, 14. J6: IR (film) 3420, 2970, 2920, 1720. 1490). 1450. 1370. 1300. $1260 \mathrm{~cm}^{-1}$. MS (Cl) calcd for $\mathrm{C}_{12} \mathrm{H}_{16} \mathrm{O}_{3}$ $[\mathrm{M}+\mathrm{H}] ' 209$. found 209 .

Ethyll 3-hyddroxy-4-methỵlpentanoate (4) ${ }^{\mathrm{l}} \mathrm{H}$ NMR (400) $\left.\mathrm{MH} / . \mathrm{CDCl}_{3}\right) \delta 4.17(2 \mathrm{H} . \mathrm{q} . J=7.1+\mathrm{H} / 2.3 .78(\mathrm{lH} . \mathrm{m}) .3 .05$ (1H. br s). 2.50 (l 1 H. dd. $J=2.92 .2 .92 \mathrm{H} / 2.2 .40(1 \mathrm{H} . \mathrm{dd} . J=$ $9.53 .9 .53 \mathrm{H} \%) .1 .72(\mathrm{lH} . \mathrm{m}) .1 .27(3 \mathrm{H} .1 . J=7.15 \mathrm{H} / .0 .94$ $(6 \mathrm{H} . \mathrm{q} . J=5.99 \mathrm{H} / 2) .{ }^{13} \mathrm{C} \mathrm{NMR}\left(100 \mathrm{MH} \% \mathrm{CDCl}_{3}\right) \delta 173.86$. $73.09 .61 .05,38.84,33.52 .18 .70 .18,10,14.52:$ IR (film) 3440. 2975.1720. 1470.1370. 1320.1270 $\mathrm{cm}^{-1}$ : MS (Cl) calcd for $\mathrm{C}_{8} \mathrm{H}_{17} \mathrm{O}_{3}|\mathrm{M}+\mathrm{H}| \cdot \mathrm{l} \mid 6 \mathrm{l}$, found $\mathrm{l} 6 \mathrm{l}$.

Ethyl 3-cyclohexyl-3-hydroxypropanoate (5) 'H NMR (400 MH\%. CDCl $) \delta 4.17$ (2H. q). 3.79)-3.75 (l H. m). 2.84 (lH. br s). $2.51(\mathrm{lH} . \mathrm{dd} . J=2.80 .2 .86 \mathrm{H} / 2) .2 .4 \mathrm{l}(\mathrm{lH} . \mathrm{dd} . J=$ 9.50. $9.49 \mathrm{H} /$ ). $1.86(1 \mathrm{H} . \mathrm{d} . J=12.65 \mathrm{H} /) .1 .76(2 \mathrm{H} . \mathrm{m})$. $1.66(2 \mathrm{H} . \mathrm{d} . J=12.30 \mathrm{H} \%) .1 .41-1.11(9 \mathrm{H} . \mathrm{m}):{ }^{13} \mathrm{C}$ NMR (100) $\left.\mathrm{MH} \%, \mathrm{CDCl}_{3}\right) \delta 174,60,73,17,61,69,44,07.39 .59$. 29.80, 29,27, 27.44, 27.18, 27.06, 15.20: IR (film) 3500 . $3050.2980 .2920,2840,1720,1+50,1410,1370,1260 \mathrm{~cm}^{-1}$. MS (Cl) calcd for $\mathrm{C}_{11} \mathrm{H}_{2\rfloor} \mathrm{O}_{3}[\mathrm{M}+\mathrm{H}]^{\prime} 201$. found 20$]$.

Ethyl 3-hydroxy-3-(p-methỵlphenyl)propanoate (7) ${ }^{1} \mathrm{H}$ NMR $\left.(400) \mathrm{MH} \% . \mathrm{CDCl}_{3}\right) \delta 7.25(2 \mathrm{H}$. d. $J=8.02 \mathrm{H} / 2.7 .14$ $(2 \mathrm{H} . \mathrm{d} . J=7.95 \mathrm{H} /) .5 .08(\mathrm{lH} . \mathrm{q} . J=4.18 \mathrm{H} /) .4 .16(2 \mathrm{H} . \mathrm{q}$. $J=7.13 \mathrm{H} \%) .3 .1(1 \mathrm{H}$. br s). $2.77-2.57(2 \mathrm{H} . \mathrm{m}) .2 .33(3 \mathrm{H} . \mathrm{s})$. $1.25(3 \mathrm{H} .1 . J=7.1+\mathrm{H} \%):{ }^{13} \mathrm{C}$ NMR $\left(100 \mathrm{MH} \% . \mathrm{CDCl}_{3}\right) \delta$ 172.80. 140.04. 137.84. 129,58. 126,02.70,57.61,21. 43.76. 21.49. 14.54: IR (film) 3410, 2960, 2900, 1715. 1510. 1440 . 1390. 1365.1290.1260 $\mathrm{cm}^{-1}$ : MS (CI) calcd for $\mathrm{C}_{12} \mathrm{H}_{17} \mathrm{O}_{3}$ $[\mathrm{M}+\mathrm{H}]^{+} 209$, found 209 .

Ethyl 3-(p-chlorophenyl)-3-hydroxypropanoate (8) ${ }^{1} \mathrm{H}$ NMR $\left(400 \mathrm{MHz}, \mathrm{CDCl}_{3}\right) \delta 7.31(4 \mathrm{H} . \mathrm{s}), 5.09(1 \mathrm{H}, \mathrm{q} . J=$ $4.25 \mathrm{~Hz}), 4.17(2 \mathrm{H}$. q. $J=7.12 \mathrm{~Hz}), 3.22(1 \mathrm{H}$. br s). 2.68 $(2 \mathrm{H}, \mathrm{m}) .1 .25(3 \mathrm{H} . \mathrm{t} . J=7.10 \mathrm{~Hz}) ;{ }^{1.3} \mathrm{C}$ NMR $(100 \mathrm{MHz}$. $\left.\mathrm{CDCl}_{3}\right) \delta 172.24 .141 .03,133.45 .128 .67 .127 .09,69.62$. 61.00. 43.20. 14.13: IR (film) 3400. 2930. 2860. 1695, 1470 . 1375. 1350. 1280. 1260.1240 $\mathrm{cm}^{-1}$ : MS (CI) calcd for $\mathrm{C}_{11} \mathrm{H}_{1}+\mathrm{ClO}_{3}[\mathrm{M}+\mathrm{H}\rceil^{-} 229$. found 229 .

Ethyl 3-(m-bromophenyl)-3-hydroxypropanoate (9) ${ }^{1} \mathrm{H}$ NMR $\left(400 \mathrm{MHz}, \mathrm{CDCl}_{3}\right) \delta 7.55(1 \mathrm{H}, \mathrm{t} . J=1.62 \mathrm{~Hz}) .7 .42-$ $7.40(1 \mathrm{H} . \mathrm{m}) .7 .30-7.19(2 \mathrm{H} . \mathrm{m}) .5 .08(1 \mathrm{H} . \mathrm{m}) .4 .18(2 \mathrm{H} . \mathrm{q}$. $J=7.14 \mathrm{~Hz}) .3 .41(1 \mathrm{H} . \mathrm{d} . J=3.55 \mathrm{~Hz}) .2 .71(2 \mathrm{H} . \mathrm{d} . J=6.84$ $\mathrm{Hz}) .1 .26(3 \mathrm{H} . \mathrm{t}, J=7.17 \mathrm{~Hz}):{ }^{1.3} \mathrm{C} \mathrm{NMR}\left(100 \mathrm{MHz}, \mathrm{CDCl}_{3}\right)$ $\delta 172.23,144.78,130.84,130.12 .128 .87,124.28,122.67$. 69.59. 61.04. 43.14, 14.14: IR (film) 3500, 3050, 2980. 1720. $1420.1260 \mathrm{~cm}^{-1}$ : MS (CI) caled for $\mathrm{C}_{11} \mathrm{H}_{1+} \mathrm{BrO}_{3}$ $\left\lceil\mathrm{M}+\left.\mathrm{H}\right|^{+} 273\right.$, found 273 .
Ethyl 3-hydroxy-3-(p-methoxyphenyl)propanoate (10) ${ }^{\mathrm{l}} \mathrm{H}$ NMR $\left(400 \mathrm{MH} / . \mathrm{CDCl}_{3}\right) \delta 7.30(2 \mathrm{H} . \mathrm{d} . J=8.09 \mathrm{H} / 2)$. $6.89(2 \mathrm{H} . \mathrm{d} . J=8.49 \mathrm{H} \%) .5 .08(1 \mathrm{H}, \mathrm{t} . J=4.53 \mathrm{H} \%) .4 .18$ $(2 \mathrm{H}, \mathrm{q} . J=7.14 \mathrm{H} /), 3.79(3 \mathrm{H}, \mathrm{s}) .3 .20(\mathrm{lH}, \mathrm{br} \mathrm{s}) .2 .78-2.64$ $(2 \mathrm{H}, \mathrm{m}) .1 .25(3 \mathrm{H}, 1, J=7.38 \mathrm{H} / 2):{ }^{13} \mathrm{C}$ NMR $(100 \mathrm{MH} \%$ $\left.\mathrm{CDCl}_{3}\right) \delta 172.44,159.20 .134 .73,126.97 .113 .91 .69 .97$. $60.83,55.29,43.33,14,16$ : IR (lilm) 3450, 2970, 2830. 1715. 1600.1500. 1460.1365.1295.1240 $\mathrm{cm}^{-1}: \mathrm{MS}(\mathrm{Cl})$ calcd for $\mathrm{C}_{12} \mathrm{H}_{1}, \mathrm{O}_{4}[\mathrm{M}+\mathrm{H}] 255$, found 255 .

Ethyl 3-hydroxy-3-(2,6-dimethoxyphenyl)propanoate (11) ${ }^{\mathrm{H}} \mathrm{H} \mathrm{NMR}\left(400 \mathrm{MH} /, \mathrm{CDCl}_{3}\right) \delta 7.19(\mathrm{lH}, \mathrm{t} . J=8.3+\mathrm{H} z)$. $6.56(2 \mathrm{H}, \mathrm{d} . J=8.41 \mathrm{H} /) .5 .66(\mathrm{lH} . \mathrm{dd} . J=4.21 .4 .3 \mathrm{l} \mathrm{H} \%)$. $4.16(2 \mathrm{H}, \mathrm{q} . J=7.14 \mathrm{H} /) .3 .84(6 \mathrm{H}, \mathrm{s}) .3 .55(\mathrm{lH}, \mathrm{br} \mathrm{s}) .2 .99$ (lH. dd. $J=9.87 .9 .91 \mathrm{H} /$ ). $2.65(1 \mathrm{H}$. dd. $J=4.25 .4 .36 \mathrm{H} / 2$ ). $1.26(3 \mathrm{H} . \mathrm{t} . J=7.1 \mathrm{H} \%):{ }^{13} \mathrm{C}$ NMR $\left(100 \mathrm{MH} \% \mathrm{CDCl}_{3}\right) \delta$ $172,01.158,07,129,30,118,40,104.59,64,94,60.79,56.10$. 42.74, 14.60: IR (film) 33525, 2960, 2920, 2820. 1720. 1580. $1460.1365 .1260 \mathrm{~cm}^{-1}$ : MS (Cl) calcd for $\mathrm{C}_{13} \mathrm{H}_{10} \mathrm{O}_{5}$ $[\mathrm{M}+\mathrm{H}] 255$, found 255 .

Ethyl 3-hydroxy-3-(o-nitrophenyl)propanoate (12) ${ }^{1} \mathrm{H}$ $\operatorname{NMR}\left(400 \mathrm{MH} \% \mathrm{CDCl}_{3}\right) \delta 7.95(\mathrm{dd} . J=0.95,1.15 \mathrm{H} \% \mathrm{lH})$. $7.89(\mathrm{dd} . J=0.76 .0,66 \mathrm{H} / . \mathrm{lH}) .7 .67(\mathrm{~m} . \mathrm{lH}) .7 .44(\mathrm{~m} . \mathrm{lH})$. $5.66(\mathrm{~d} . J=9.37 \mathrm{H} \% \mathrm{lH}) .4 .2(\mathrm{q} . J=7.14 \mathrm{H} \% .2 \mathrm{H}) .3 .86(\mathrm{~d} . J$ $=2.59 \mathrm{H} \% \mathrm{lH}) .2 .96(\mathrm{dd} . J=2.76 .2 .76 \mathrm{H} \% \mathrm{lH}) \cdot 2.66(\mathrm{dd} . J$ $=9.4 .9 .36 \mathrm{H} \% \mathrm{IH}) .1 .27(\mathrm{t} . J=7.12 \mathrm{H} \% 3 \mathrm{H}):{ }^{13} \mathrm{C}$ NMR (100 $\left.\mathrm{MH} / . \mathrm{CDCl}_{3}\right) \delta 172.66 .147 .73 .138 .5 .134 .16 .128 .83$. 128.54. 124.83. 66.33. 61.5. 42.78. 14.49: IR (film) 3420. $2960.1700 .1500 .1320 .1280 .1250 \mathrm{~cm}^{-1}$. MS (Cl) calcd for $\mathrm{C}_{11} \mathrm{H}_{13} \mathrm{NO} \mathrm{O}_{5}[\mathrm{M}+\mathrm{H}] 240$. found 240 .

Ethyl 3-hydroxy-3-(o-hydroxyphenyl)propanoate (13) ${ }^{1} \mathrm{H}$ NMR $\left(400 \mathrm{MH} \% \mathrm{CDCl}_{3}\right) \delta 7.18(\mathrm{lH} . \mathrm{m}) .6 .98(\mathrm{lH} . \mathrm{dd} . J$ $=1.43 .1 .43 \mathrm{H} /$ ) $.6 .88-6,8 \mathrm{l}(2 \mathrm{H} . \mathrm{m}) .5 .27$ (lH. dd. $J=3.06$. $3.06 \mathrm{H} /$ ). $4.2 \mathrm{l}(2 \mathrm{H} . \mathrm{q} . J=7.14 \mathrm{H} /$ ). $2.95(\mathrm{lH} . \mathrm{dd} . J=10.27$. $10.25 \mathrm{H} /) .2 .72(\mathrm{lH} . \mathrm{dd} . J=3.16 .3 .1 \mathrm{I} \mathrm{H} /) .1 .28(3 \mathrm{H} . \mathrm{t} . J=$ $7.15 \mathrm{H} \%$ ): ${ }^{13} \mathrm{C}$ NMR (100 MH\%. $\left.\mathrm{CDCl}_{3}\right) \delta 173.08 .155 .66$. 129.32. 126.66. 125.31. 119.97. 117.48.71.48.61.28. 40.95. 14.11: IR (film) 3370. 2980. 1700. 1610. 1490. 1450. 1370. 1350. $1220 \mathrm{~cm}^{-1}$ : MS (CI) calcd for $\mathrm{C}_{11} \mathrm{H}_{15} \mathrm{O}_{4}[\mathrm{M}+\mathrm{H}]^{+} 211$. found 211 .

Ethyl 3-hydroxy-3-(m-hydroxyphenyl)propanoate (14) ${ }^{1} \mathrm{H} \mathrm{NMR}\left(400 \mathrm{MHz} . \mathrm{CDCl}_{3}\right) \delta 7.15(1 \mathrm{H} . \mathrm{t} . J=7.8 \mathrm{~Hz}) .6 .83$ (1H. d. $J=7.08 \mathrm{~Hz}) .6 .73(2 \mathrm{H} . \mathrm{m}), 5.05(3 \mathrm{H}, \mathrm{q} . J=4.32 \mathrm{~Hz}$, H-3). 4.14 (2H. q. $J=7.13 \mathrm{~Hz}) .2 .72(1 \mathrm{H} . \mathrm{dd} . J=9.02 .9 .02$ Hz). 2.65 (1H. dd. $J=3.95 .3 .94 \mathrm{~Hz}$ ). 1.23 (3H. t. $J=7.13$ $\mathrm{Hz}):{ }^{1.3} \mathrm{C}$ NMR (100 MHz, $\left.\mathrm{CDCl}_{3}\right) \delta 172.64 .156 .18 .143 .96$. 129.81, 117.74. 115.10. 112.77, 70.38, 61.15. 43.18. 14.07: IR (film) 3380. 3050, 2980, 1720. 1600. 1450, 1420, 1250 $\mathrm{cm}^{-1}$ : MS (CI) calcd for $\mathrm{C}_{11} \mathrm{H}_{\rfloor_{3}} \mathrm{O}_{4}[\mathrm{M}+\mathrm{H}]^{+} 211$, found 211 .

Ethyl 3-hydroxy-3-(2-furyl)propanoate (15) 'H NMR $\left(400 \mathrm{MHz}, \mathrm{CDCl}_{3}\right) \delta 7.36(\mathrm{t} . J=0.89 \mathrm{~Hz} . \mathrm{lH}) .6 .33-6.26(\mathrm{~m}$. $2 \mathrm{H}) .5 .13$ (q. $J=4.23 \mathrm{~Hz}, 1 \mathrm{H}) .4 .18(\mathrm{q} . J=7.11 \mathrm{~Hz} .2 \mathrm{H})$. 3.25 (s. lH). $2.92-2.79(\mathrm{~m} .2 \mathrm{H}) .1 .26(\mathrm{t} . J=7.16 \mathrm{~Hz} .3 \mathrm{H})$ : ${ }^{1.3} \mathrm{C}$ NMR $\left(100 \mathrm{MHz}, \mathrm{CDCl}_{3}\right) \delta 170.29 .153 .2,14.59$. 108.62, 104.65. 62.55, 59.33, 38.24, 12.5; IR (filun) 3450. 2960. 2920.1715, 1640,1500,1360. 1280. $1240 \mathrm{~cm}^{-1}$ : MS (CI) calcd for $\mathrm{C}_{6} \mathrm{H}_{12} \mathrm{O}_{4}\lceil\mathrm{M}+\mathrm{H}\rceil^{-} 185$. found 185 .

Ethyl 3-hydroxy-3-(2-thienyl)propanoate (16) 'H NMR 
$\left(400 \mathrm{MH}_{.} \mathrm{CDCl}_{3}\right) \delta 7.26-7.23(1 \mathrm{H}, \mathrm{m}) .6 .96-6.94(2 \mathrm{H}, \mathrm{m})$. $5.36(1 \mathrm{H}, \mathrm{m}) .4 .58(2 \mathrm{H}, \mathrm{q} . J=7.14 \mathrm{H} / \mathrm{s}) .3 .55(\mathrm{lH}, \mathrm{d} J=4.27$ $\mathrm{H} /) .29\left(0-2.79(1 \mathrm{H}, \mathrm{m}) .1 .26(3 \mathrm{H}, 1 . j=7.2 \mathrm{H} \%) .{ }^{13} \mathrm{C} \mathrm{NMR}\right.$ (100 $\left.\mathrm{MH} / \ldots \mathrm{CDCl}_{3}\right) \delta 172.32,146.78,127.1,125.24,124.03$. 66.95. 61.39. 43,61, 14.53: $\mathrm{MS}(\mathrm{Cl})$ calcd for $\mathrm{C}_{9} \mathrm{H}_{13} \mathrm{O}_{3} \mathrm{~S}$ $[\mathrm{M}+\mathrm{H}]$ ' 20], found 20].

Ethỵl 3-hydroxy-5-phenyl-t-pentenoate (17) ${ }^{1} \mathrm{H}$ NMR (400 $\left.\mathrm{MH} / . . \mathrm{CDCl}_{3}\right) \delta 7.38-7.2 \mathrm{l}(\mathrm{m} .5 \mathrm{H}) .6 .65$ (d. $J=16.04$ $\mathrm{H} / \mathrm{lH}) .6 .22(\mathrm{dd} . J=6.12 \mathrm{H} / . \mathrm{lH}) .4 .72(\mathrm{~m} . \mathrm{lH}), 4.18(\mathrm{q} . J$ $=7.15 \mathrm{H} / .2 \mathrm{H}) .2 .95(\mathrm{~s} . \mathrm{lH}) .2 .68-2.57(\mathrm{~m}, 2 \mathrm{H}) .1 .27(1 . J=$ $7.17 \mathrm{H} / .3 \mathrm{H}):{ }^{13} \mathrm{C}$ NMR $\left(100 \mathrm{MH} / . \mathrm{CDCl}_{3}\right) \delta 172.65$. 136.85. 131.16. 130.37, 128.98. 128.21. 126.95, 69.29. 61.27.41.93. 14.5\%: IR (film) 3400, 2\%60. 1710. 1490. 1440. 1380. 1360. $1270.1230 \mathrm{~cm}^{-1}$. MS (Cl) calcd for $\mathrm{C}_{13} \mathrm{H}_{16} \mathrm{O}_{3}$ $[\mathrm{M}+\mathrm{H}]^{\prime} 22 \mathrm{l}$. found $22 \mathrm{l}$.

Ethyl 3-hyddroxy-3-methylbutanoate (18) ${ }^{\mathrm{H}} \mathrm{H}$ NMR (400) $\left.\mathrm{MH} \% \mathrm{CDCl}_{3}\right) \delta 4.18$ (q. $J=7.16 \mathrm{H} \% 2 \mathrm{H}$ ). $3.6(\mathrm{~s} . \mathrm{lH}) .2 .48$ (s. $2 \mathrm{H}) .1 .3-1.25(\mathrm{~m} .9 \mathrm{H}) \cdot{ }^{13} \mathrm{C}$ NMR $\left(100 \mathrm{MH} \% \mathrm{CDCl}_{3}\right) \delta$ 173.04. 69.00, 60.64. 46.31.29.14. 14.19: IR (film) 3440. 2960. 2910. 1710. 1370. $1200 \mathrm{~cm}^{-1}$. MS (CI) calcd for $\mathrm{C}_{7} \mathrm{H}_{14} \mathrm{O}_{3}[\mathrm{M}+\mathrm{H}]$ ' 147. found $\mathrm{l}+7$.

1-Ethoxycarbonyylmethỵlcyclohexanol (19) ${ }^{\mathrm{H}} \mathrm{H}$ NMR (400 $\left.\mathrm{MH} \approx . \mathrm{CDCl}_{3}\right) \delta 4.17$ (q. $\left.J=7.14 \mathrm{H} \% .2 \mathrm{H}\right) .3 .44(\mathrm{~s} . \mathrm{HH}$ ). 2.46 (s. 2H). 1.72-1.62 (m. 4H). 1.56-1.47 (m. IH). 1.46$1.32(\mathrm{~m}, 4 \mathrm{H}), 1.27(\mathrm{~m}, 4 \mathrm{H}):{ }^{13} \mathrm{C}$ NMR $(\mathrm{l} 00 \mathrm{MH} \% \mathrm{CDCl}) \delta$ 173.32. 70.31.60.92. 45.65. 37.80. 25.98. 22.36. 14.56: IR (film) $3500,2910,2850,1705,1450,1370,1310,1230$ $\mathrm{cm}^{-1}$. MS (CI) calcd for $\mathrm{C}_{101} \mathrm{H}_{13} \mathrm{O}_{3}[\mathrm{M}+\mathrm{H}]$ 187. Found 187.

Ethyl 3-hyddroxy-3-phenylbutanoate (20) ${ }^{\prime} \mathrm{H}$ NMR (400) $\left.\mathrm{MH} \nsim \mathrm{CDCl}_{3}\right) \delta 7,43(2 \mathrm{H}, \mathrm{m}) .7,33(2 \mathrm{H}, \mathrm{m}) .7,25-7,2 \mathrm{l}(\mathrm{lH}$. m). 4.39 (l H. s). $4.05(2 \mathrm{H}$. q. $J=7.13 \mathrm{H} / 2.2 .88(2 \mathrm{H} . \mathrm{dd} . J=$ 15.8. $15.9 \mathrm{H} / 2) .1 .54(3 \mathrm{H}, \mathrm{s}) .1 .12(3 \mathrm{H} .1 . J=7.2 \mathrm{H} /)^{13} \mathrm{C}$ NMR (100 MH $\left.\% \mathrm{CDCl}_{\S}\right) \delta 172.72,146,83,128,23,126.84$. 124.45. 72.74. 60.73. 46,41, 30.66, 13.98: IR (film) 3460. 3020. 2960.2900. 1690. 1480. 1430. 1360. 1320. $1240 \mathrm{~cm}^{-1}$. MS (CI) calcd for $\mathrm{C}_{12} \mathrm{H}_{17} \mathrm{O}_{3}[\mathrm{M}+\mathrm{H}]^{\prime} 209$. found 209 ,

Ethyl 3-hydroxy-3-(p-methylphenyl)propanoate (21) ${ }^{1} \mathrm{H}$ NMR (400 MHz, $\left.\mathrm{CDCl}_{3}\right) \delta 7.43$ (m. 2H), 7.33 (m. 2H), $7.25-7.21(\mathrm{~m} .1 \mathrm{H}), 4.39$ (s. 1H) 4.05 (q. $J=7.13 \mathrm{~Hz} .2 \mathrm{H}$ ). $2.88($ dd $J=15.8 .15 .9 \mathrm{~Hz}, 2 \mathrm{H}), 1.54$ (s. $3 \mathrm{H}) .1 .12(\mathrm{t} . J=7.2$ $\mathrm{Hz}, 3 \mathrm{H}) ;{ }^{13} \mathrm{C}$ NMR $\left(100 \mathrm{MHz} . \mathrm{CDCl}_{3}\right) \delta 172.72,146.83$. $128.23,126.84,124.45,72.74,60.73,46.41,30.66,13.98$ : IR (film) 3460, 3020. 2960, 2900. 1690, 1480. 1430. 1360 . 1320. $1240 \mathrm{~cm}^{-1}$ : MS (CI) calcd for $\mathrm{C}_{1} \mathrm{H}_{16} \mathrm{O}_{3}[\mathrm{M}+\mathrm{H}]^{-} 222$. found 222

Ethyl 3-hydroxy-2-methyl-3-phenylpropanoate (22) ${ }^{1} \mathrm{H}$ NMR (200 MHz, $\left.\mathrm{CDCl}_{3}\right) \delta 7.35(\mathrm{~m} .5 \mathrm{H}), 5.01(\mathrm{t} . J=4.63$ Hz $1 \mathrm{H}) .3 .70(\mathrm{~d} . J=11.90 \mathrm{~Hz} .3 \mathrm{H}), 3.01(\mathrm{~m}, 1 \mathrm{H}) .2 .82(\mathrm{~m}$. 1H). $1.11(\mathrm{~d}, J=7.33 \mathrm{~Hz}, 3 \mathrm{H})$.

3-Hydroxy-2,2,-dimethyl-3-phenylpropanoate (23) ${ }^{1} \mathrm{H}$ NMR (200 MHz. CDCl 3 ) $\delta 7.31$ (m. $5 \mathrm{H}) .4 .91$ (d. $J=4.27$ Hz. $1 \mathrm{H}) .4 .17(\mathrm{q} . J=7.01 \mathrm{~Hz} .2 \mathrm{H}) .3 .18(\mathrm{~d} . J=4.17 \mathrm{~Hz} .1 \mathrm{H})$. $1.27(\mathrm{t} . J=7.01 \mathrm{~Hz}, 3 \mathrm{H}) .1 .13(\mathrm{~d} . J=5.19 \mathrm{~Hz}, 6 \mathrm{H})$.
Acknowledgment. This work was supported by grant No. (R02-2002-000)-(00) (0)6-(0) from the Basic Research Program of the Korea Science \& Engineering Foundation. The gas chromatograms were provided by GC facility. supported by Rescarch Center for Advanced Mincral Aggregate Composite Products.

\section{References}

1. (a) Shriner, R. I. Org. React. 1946, 1, 1. (b) Rathke. M. W. Org. React 1975, 22,423. (c) Gensler. W. J. Chem Rey 1957, 57,265. (d) Diaper. I). (i. M.: Kuksis. A. (hem. Ror 1959. 59.89.

2. (a) Becher. D.: Brodsky. N.: Kalo. I. J. Org. (hem. 1978. 43. 2557. (b) Barco. A.: Benett. S.: Pollini. G. P. J. Org (hem. 1980. 45. 4776. (c) Klipa. D. K.: Hart. H. J. Org. Chem. 1981. 46. 2815. (d) West. H: (i.: Gunatuardena. (i. V. J. Org. Chom 1993, 58.5043. (e) Witzemamm, I. S.: Nottinghem. W. I). f. Org ( Chem. 1989. 54 3258 and relerences cited therein

3. (a) March. T. Adromed Orgamic (hemistry: Wiley: New York. 1992: p 929. (b) Marioka. K.: Hashimoto. S.: Kitagawa. Y.: Yamamoto. H.: Nozaki. H. J. im (Chem. Soc. 1977. 99.7705. (c) Ilarada. T.: Mukaiyame. T. Chem. Lett 1982. 16l. (d) Imamoto. T:: Kusumoto. $\mathrm{l}$ : Tanarayama, Y.: Sugiura. Y: Yokovama. M. $d$.

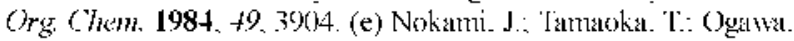
H.: Wakabayashi. S. Chem. Lett. 1986. 541. (t) Shen. Z.: Zhang. J.: Zoul. H.: Yang. M. Tefrahedron Leth 1997. 38. 2733. (o) Gabricl. T.: Wessjohann. L. Tetrohedron Lett. 1997. 38. 1363. (h) Gabriel. T: Wessjohann. I. Fetrahedron Itett. 1947, 38,4387. (i) Chao. I..C. Rieke R. I) J. Org (Chem. 1975. 40.2253. (i) Araki.

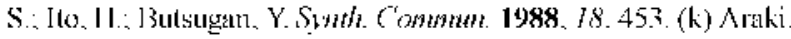
S.: Ito. H.: Katsumura. N.: Butsugan. Y. J. Orgomomet. Chem. 1989. 369. 291. (I) Araki. S.: Katsumura. N.: Kawasaki. K.: Butsugan. Y. I. Chem. Soc. Perhin Trans. I 1991. 499. (m) Johar. P. S.: Araki. S.: Butsugan. Y. J. (Them. Soc. Pethin Trans. 1992. 7II. (n) Schick. I.: I udduig. R.: Schwars. K.-II: Kleiner. K.

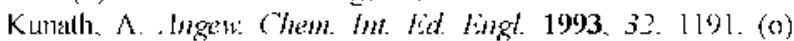
Cahicz. G.: Chavant. P.-Y. Tetwedrom Le'th 1989. 30. 7373. (p) Kagan. H. B.: Namy. I.-L.: Girard. P. Tetwhedron 1981. 37. 175. (q) Tabuchi. T: Kawamura. K.: Inanaga. I.: Yamaguchi. M. Fotrahedon hett 1986, 27,3889 . (r) Molander. G. A. Filler. I. I3 J. im Chem. Soc. 1987. 109, 6556. (s) I Iuang. X.: Xie. I.: Wu, II Tetrahedon tent 1987. 28. 801

4. (a) Fustner. A. Sinthesis 1989. 571 . (b) Fustner. A. Angent Chem. If7. Ed. Engl 1993. 32. 164.

5. Ricke. R. D.: Ulm. S. I. Synthesis 1975.452.

6. (a) Mason. I. I.: Jorimer. I. P. Sonochenistry: Theory: ipplications and t ses of thasound in Chemistry lillis I forwood I. imited: Chichester. I988. (b) I Ian. 13. II.: I3oudjouk. P. J. Org (7rm. 1982. t7. 5030 . (c) Nishiyama. T.: Woodhall. .T. F.: Lawson. E. N.: Kitching. W. J. (hrg. (Thm 1989. 54. 2184.

7. (a) Lce. P. H:: Bang. K:: Loc. K.: Lcc. C.-H.: Chang. S. Fetrahedon leth 20MO. H, 752I. (b) I.ee. P. I I.: I3ang. K.: Ahn. II.: J.ee. K. Bulf Sorem Chem. Soc. 2001. 22, 1385. (c) I ee. P.

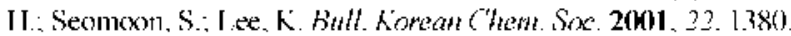
(d) Lec. P. H.: Lec. K.: Sung. S.-Y. Chang. S. J. Org. Chem. 2001. 66. 8646. (c) Lec. P. H.: Lee. K.: Kim. S. Org. Lett. 2001. 3. 3205. (f) Lee. P. H.: Sung. S.-Y.: Lcc. K. Ong. Lett. 2001. 3. 3201. (g)

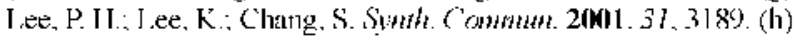
I.ee, P. H.; Aht, II: T.ee, K.; Sung. S.-Y: Kim. S. Tetrahedon lent 2001, 42, 37. (i) I ee. P. H.: Sung. S.-Y.: T.ee, K.: Chang. S. Silfhlett 2002. 146.

8. Lec. P. H.: Bang. K.: Lcc. K.: Sung. S.-Y.: Chang. S. Symh. Commm. 2001.31.3781. 